\title{
Automation for Genomics, Part One: Preparation for Sequencing
}

\author{
Deirdre Meldrum ${ }^{1}$
}

Department of Electrical Engineering, Genomation Laboratory, University of Washington, Seattle, Washington 98195-2500 USA

In the past four years, automation for genomics has enabled a 43-fold increase in the total finished human genomic sequence in the world. This two-part noncomprehensive review will provide an overview of different types of automation equipment used in genome sequencing. Part One focuses on equipment involved in DNA preparation, DNA sequencing reactions, and other automated procedures for preparing DNA for running on sequencers or subsequent analysis; it also includes information on the development of these machines at various genome centers. Part Two, to be published in the next issue, will cover sequencing machinery and array technology, and conclude with a look at the future technologies that will revolutionize molecular biology. "Alternate" sequencing technologies (including mass spectrometry, biochips, and single-molecule analysis) will also be examined.

New advances in medicine are being made at a rapidly increasing pace, largely due to recent developments in genome analysis. DNA sequence analysis provides a clearer understanding of how genetic variation leads to disease, and thus will ultimately lead to new cures. Three major milestones - the invention of sequencing reactions (Maxam and Gilbert 1977; Sanger et al. 1977), the Polymerase Chain Reaction (PCR) (Mullis et al. 1986; Mullis and Faloona 1987), and automated fluorescent DNA sequencers (Smith et al. 1985, 1986; Hood et al. 1987; Hunkapiller et al. 1991) — have made it possible to streamline and automate most of the processes required for DNA analysis.

Today there are instruments that can automate nearly every step of the large-scale sequencing process: isolating DNA, cloning or amplifying DNA, preparing enzymatic sequencing reactions, purifying DNA, and separating and detecting DNA fragments with fluorescent labels to obtain DNA sequence. Most large-scale sequencing facilities employ the consensus strategy as outlined in Waterston and Sulston (1998), which combines a random shotgun phase with a directed finishing phase. Other centers use a whole-genome shotgun approach: random fragments of total human genomic DNA are subcloned directly, and high-throughput sequencing is used to generate random sequences that provide at least ten-fold coverage of the genome (Venter et al. 1998; Waterston and Sulston 1998). Yet another approach is to sequence each end of every BAC in a genomic library. Matching a finished sequence to one or more BAC ends permits selection of the net clone (Venter et al. 1996; Waterston and Sulston 1998).

Most genome centers use a combination of

'E-MAIL deedee@ee.washington.edu; FAX (206) 221-5264. Article and publication are at www.genome.org/cgi/doi/10.1101/gr. 101400 manual methods, in-house automation, and commercial automation (Jandreski 1995; Wendl et al. 1998; Marshall 1999; Mullikin and McMurragy 1999; Scientific American 2000; Spurr et al. 1999; Swanson 2000; Watson et al. 1993). Each center is unique in how it is organized and how the sequencing data is obtained. When some of the emerging chip-based technologies reach maturity, we may one day see true "laboratorieson-a-chip" that will be capable of taking a DNA sample and carrying it through the entire analytical process. Although the current cost of sequencing is \$0.20$\$ 0.30 /$ base of high quality (less than one error in 10,000 bases) finished sequence (http://www.nhgri. nih.gov/NEWS/pilot_project_completion.html), and new technologies continue to push this price lower and lower.

To meet the growing demand for genomic sequencing and analysis, "there is a need for individuals who are highly trained in the interdisciplinary sciences related to genome research" (Roberts 1993). Biologists, engineers, and scientists have recently been working together in order to leverage their expertise. The new technologies developed as a result of these crossdisciplinary interactions are producing a revolution in the way biology is performed and analyzed. Advances in biology are driving technology, and conversely, advances in technology are driving biology (Meldrum 1995).

We are now seeing an exponential growth in the number of sequencing experiments being performed and the volume of data collected. This growth, driven by the Human Genome Project (Collins et al. 1998) and other large-scale sequencing projects, is made possible by advances in sequencing methods and laboratory automation (for details on informatics for genome analysis, see Burks 1994, Goodman 1996, White and 
Kerlavage 1996, Smith et al. 1997, and the March 1998 Genome Research special informatics issue). Discussions of automation equipment, including its development and future technologies, is presented below and in the second part of this review (next issue). See the websites referenced for photos or further details on the instruments discussed.

\section{Large-Scale Sequencing Goals and Automation Needs}

On June 26, 2000, a landmark achievement was announced: the compilation of a working draft of the human genome by the Human Genome Project, and Celera Genomics' (Rockville, MD) first assembly of a complete human genome sequence (Macilwain 2000; http://www.nhgri.nih.gov/news/sequencing_consortium.html). The types of automation used for largescale sequencing at a variety of public and private genome centers can vary dramatically, both in the machines and robots used. The current automation systems being utilized by sequencing centers have been considered evolutionary rather than revolutionary in nature. The centers have chosen to include discrete instruments to automate only certain parts of their processes as necessary to meet their sequencing goals. These instruments include both in-house developments and commercially available systems that largely imitate the steps that would be performed in a manual process. They provide an improvement in reliability and throughput as well as a decrease in costs. The throughput of these centers has increased substantially during the past two years. In fact, the world total of finished human genomic sequence increased from 15 to $180 \mathrm{Mb}$ between 1996 and 1998 (Waterston and Sulston 1998) and from $186 \mathrm{Mb}$ to $645 \mathrm{Mb}$ between February 1998 and June 2000. Draft sequence went from $146 \mathrm{Mb}$ to $2938 \mathrm{Mb}$ between October 1998 and June 2000, producing a 12-fold increase in just the last year (http://www.ncbi.nlm.nih.gov/genome/seq/ HsHome.shtml). The working draft sequence, defined as on average at least $4 \mathrm{x}$ coverage in at least Q20 bases (and not less than $3 x$ coverage for any clone), has been the primary emphasis in the past year. Most of the draft sequence is now at the working-draft standard. This trend in sequencing productivity will continue to rise sharply as centers become more efficient at largescale sequencing and benefit from the introduction of new technology. One instrument that is currently making a substantial impact on sequencing efforts is the capillary sequencer; more information on this type of instrumentation will appear in the second part of this review.

There is a tremendous variety of systems available for genome automation at this time. Each, of course, must be carefully chosen to match the processes and throughputs required in a particular setting. The fol- lowing sections provide examples of automation systems available for various steps in large-scale sequencing, including oligo synthesizers, DNA shearing devices, plaque and colony pickers, gridders, DNA template preparation systems, sequencing reaction robotics, thermal cyclers, and multi-purpose automated workstations. Part Two of this review will cover sequencers and gel loaders as well as methods and systems for preparing microarrays. Neither part provides an exhaustive list but does give a broad view of the types of instrumentation currently available.

\section{Oligo Synthesizers}

Oligonucleotide synthesizers based on phosphoramidite chemistry have been developed to automate and increase the production of oligo production. The MerMade oligodeoxyribonucleotide synthesizer (Rayner et al. 1998) system, designed and developed at the University of Texas Southwestern Medical Center (UTSW) Genome Center (Dallas, Texas), synthesizes two 96-well plates of oligonucleotides in a single run of 17 hours using standard phosphoramidite chemistry. This system is similar to the oligo synthesizer built by the Human Genome Center at Lawrence Livermore National Lab (Sindelar and Jaklevic 1995) that processes 12 oligos/run, and the AMOS oligo synthesizer designed and built at the Stanford University Genome Center (Lashkari et al. 1995) that synthesizes 96 oligos/ 10-hr run. The MerMade is a robotic system that uses an XY table and a series of liquid control valves to produce the oligos within an Argon inert gas environment. Each well contains a glass frit to cover the port to which CPG beads have been deposited and synthesis is performed. The MerMade is controlled by a Macintoshbased Labview program with 55 virtual instruments. During the last four years over 130,000 oligos have been made at UTSW with four MerMades, at \$0.08/ base. The MerMade is now available from Bioautomation (Dallas, Texas). There are about 20 units in use throughout the world (H. Garner, pers. comm.).

Intelligent Bio-Instruments (http:// www.intelligentbio.com), a division of Intelligent Automation Systems (IAS), Inc. (Cambridge, MA) has a Primer Station 960 that is a 96-channel parallel DNA synthesizer, also based upon phosphoramidite chemistry.

GeneMachines (San Carlos, CA) (http:www.genemachines.com) designed their own 96-channel oligonucleotide synthesizer, the PolyPlex, featuring many improvements over the original AMOS machine designed at Stanford University. It is also officially licensed to use the Caruther's phosphoramidite chemistry (Scott Hunicke-Smith, pers. comm.). The PolyPlex is capable of directly generating a 96-well microplate of 20-mers in less than 3 hours. Synthesis time and reagent consumption is reduced by parallel dispensing of 
reagents with 11 dedicated reagent delivery manifolds. The cost/base is less than $\$ 0.10$.

\section{DNA Shearing}

GeneMachines' HydroShear (Thorstenson et al. 1998) uses hydrodynamic shearing forces to fragment DNA for shotgun sequencing. DNA in any concentration in volumes as small as $60 \mu \mathrm{L}$ without dilution can be processed.

Genoscope (Evry, France) (http://www.genoscope.cns.fr) constructed a small DNA shearing machine to increase DNA recovery from Qiagen columns. This machine has 96 syringes in a microtiter plate format that permits them to perform simultaneous and uniform shearing of a microplate of samples (G. Gyapay, pers. comm.).

\section{Plaque and Colony Pickers, Gridders}

The LBNL Colony Picker (Uber et al. 1991) was one of the first automated systems to combine a HewlettPackard (HP) robot arm with a charge coupled device (CCD) video camera for imaging. It could process 1920 colonies in a single run. Based upon similar technology are the Washington University Genome Sequencing Center (WashU GSC) (http://genome.wustl.edu/gsc) plaque picker (Panussis et al. 1996) and the Stanford University DNA Sequencing and Technology Center (http://sequence-www.stanford.edu) plaque picker (Marziali et al. 1999). The WashU GSC automated plaque picking and arraying robot, with a picking rate of 800 plaques/hour, identifies M13 plaques with a monochrome CCD camera and picks and arrays selected plaques using disposable intramedic tubing as the inoculating mechanism. Its advantages are that there are no metal probes to sterilize, and the intramedic tubing can be used to deposit plaque into dry tubes or tubes containing culture media. A user places nine round petri dishes with plaques into fixed positions; the computer-controlled system images the plaques with a CCD, picks them one at a time with intramedic tubing, and places a harvested plaque into a tube in a 96-tube box. A single plaque uses a 19-mm long intramedic (polyethylene) tubing segment, which yields about 1566 plaques/100 ft of tubing.

AutoGen (Framingham, MA) (http:// www.autogen.com) recently introduced the AutoGenesys, an automated robotic workstation based on robotic systems from Adept Technology, Inc. (San Jose, CA) and AutoGen for colony or plaque picking, library replicating and re-arraying, and membrane gridding. It can perform 3000 pick-place-sterilize cycles/hour using 96- or 384-well microplates or deep-well plates.

The Automation Partnership (Royston, UK and Medfield, MA) (http://www.autoprt.co.uk) has a BasePlate robotic system for picking (3500 colonies/hr), gridding (up to 300,000 spots/hr), plate replication (up to 3000 plates/day), and plate reformatting (up to 1500 wells/hr). It can accommodate 96-, 384-, or 1536-well microplates.

BioRobotics Ltd. (Cambridge, UK) (http:// www.biorobotics.co.uk) produces the BioPick automated colony picking robot that can pick up to 20,000 colonies/day with a picking accuracy of $50 \mu \mathrm{m}$ using 24-, 96- or 384-well microplates. The robot removes and replaces microplate lids, fills microplate wells with culture medium before picking, and cleans and sterilizes the needles. An optional plaque picking accessory kit is available to pick whole plugs of agar with hollow needles. BioRobotics Ltd. also produces the BioGrid for automatic gridding and library replication from 96and 384-well microplates onto a variety of membrane formats with a multi-pin tool at densities of $1 \mathrm{X}$ up to $64 X$. It can array 36,000 clones onto each of four 22$\mathrm{cm}$ membranes in $1 \mathrm{hr}$ with a density of up to 83,000 samples/membrane. Re-arraying of libraries is performed with the Total Array System (TAS) option of the BioGrid. Nanoliter volumes are transferred between microplates using a multi-pin tool.

GeneMachines Gel-2-Well instrument, based upon the Stanford University DNA Sequencing and Technology Center (http://sequence-www.stanford.edu) plaque picker (Marziali et al. 1999), is designed for picking isolated plaques and colonies. It can perform 3600 picks/hr using 96- or 384-well plates. Colonies can be picked down to $0.5-\mathrm{mm}$ diameter and color picking is an option. With 72 source plates, 72 destination plates, and 12 plate hotels that hold 12 plates each, the Gel-2-Well can run unattended for 14 hours. GeneMachines has an OmniGrid platform for gridding (to be described in the Microarraying Section in Part Two of this review).

The Genetix Ltd. (Christchurch, UK) (http:// www.genetix.co.uk) Q Pix Colony Picker picks out of $22 \times 22 \mathrm{~cm}$ bioassay trays, petri dishes or omnitrays into 96- or 384-well plates. It can pick over 3500 colonies/hr and can pick 5760 colonies unattended in under two hours. The Genetix Q Bot can also be used for colony picking. It uses a 96-pin picking head, has a capacity for 72 microplates (96- or 384-well), holds 15 filters $(22 \times 22 \mathrm{~cm})$, and picks over 3500 colonies/hr.

The Genetix Ltd. Q Bot and Q Pix both perform gridding from samples in 96- or 384-well microplates with 96 or 384 steel pin gridding heads. The Q Bot can process up to fifteen $22 \times 22 \mathrm{~cm}$ Q Filters, process up to 72 source microplates, and grid in excess of 100,000 samples/hr. The smaller Q Pix can use two filters and up to 15 source plates.

Genomic Solutions, Inc. (Ann Arbor, MI) (http:// www.genomicsolutions.com) Flexys Modular Robotic Workstations may be used for colony and plaque picking, gridding, replicating, and re-arraying. It uses a CCD camera to select colonies based on shape, size, 
and color, resulting in 99\% picking accuracy. The Flexys can grid from microplates onto nylon membranes. It uses a 24-pin pneumatic head and autoloader and can accommodate 96- or 384-well microplates.

In the Max-Planck-Institute (MPIMG) (Berlin, Germany) (http://www.mpimg-berlin-dahlem.mpg.de) production line, the MPIMG clone-picking robot employs a CCD imaging system and a 96 spring-loaded pin tool to pick 3500 clones/hour into 384-well microplates. All 96 pins are inoculated and sterilized at once. Up to two large colony trays $(225 \times 225 \mathrm{~mm})$ and twenty-four 384-well plates can be positioned on the robot bed. The replicating robot system includes stations for automated filling, barcoding, plate stacking and plate inoculation (Maier et al. 1997).

The robot used for picking at MPIMG is also used for spotting, but with a 384 spring loaded pin tool that can transfer 4-10 $\eta \mathrm{L} / 450-\mu \mathrm{m}$ diameter pin. Fifteen membrane replicates with 57,600 clones can be spotted in about three hours. Hybridizations are visualized with Attophos (JBL Scientific) a fluorescent substrate for alkaline phosphates, using automated image analysis (Maier et al. 1997).

\section{DNA Template Preparation Systems}

AutoGen produces the AutoGenprep 740, 2000, 850 Alpha, and 3000 series of automated nucleic acid extraction systems. These systems use a robotic operation with no probe or pipette tips. The AutoGenprep 740 can process up to 256 samples of plasmid, cosmid, BAC, and PAC on the same run or up to 192 samples of YAC, phage, mouse tail, plant, and buffy coat. It has the highest throughput of the AutoGen nucleic acid extraction systems. The AutoGenprep 3000 is the newest of the nucleic acid extraction systems. It can automatically isolate up to 48 samples of genomic DNA and RNA from whole blood, cultured cells, and human and animal tissue, in 4-6 hr.

At the Baylor College of Medicine-Human Genome Sequencing Center (BCM-HGSC) (http:// www.hgsc.bcm.tmc.edu), in-house robot developments have focused on the development of a device that assumes an "assembly line" mode for DNA template preparations. The protocol is based on a filter capture method and the assembly line has generated fifty 96-well plates of DNA in three hours with three technicians. The device consists of a series of plate carriers, machined slides, and in-line vacuum and dispensing stations. Plate movements are controlled by a pneumatic arm and integrated controllers. Efforts are underway to further reduce the human effort required, with the aim of building a machine that can produce one plate every two minutes, with no more than two attendees (R. Gibbs, pers. comm.).

CRS Robotics Corporation (Burlington, Ontario, Canada) (http://www.crsrobotics.com) provides stan- dard and custom robotic lab automation for drug discovery, genetic research, and clinical applications. They developed the CRS DNA Purification Workstation, based upon a design by the Department of Energy Joint Genome Institute (T. Hawkins, pers. comm.), to automate all of the reaction steps and conditions required for successful completion of the Solid-Phase Reversible Immobilization (SPRI) process (Hawkins et al. 1994; DeAngelis et al. 1995; Hawkins et al. 1997). The system uses a CRS A465 six degrees of freedom articulated robot, POLARA software, and modules for liquid handling, shaking, washing, dispensing, bead settling, lidding and de-lidding, barcode identification, plate feeding, and storage. A Robbins Hydra 96/384 well pipettor with a microplate shuttle is also used.

GeneMachines new Revolution PrepMachine originated in the laboratory of Dr. Ronald Davis at Stanford University (Marziali et al. 1999), and is designed to automate plasmid purification. The RevPrep DNA template preparation robot automates this by use of a rotary turntable, pneumatics, and an exclusive Array Centrifuge technology that has 96 individual rotors in a 96-well microplate format (Marziali, Willis, and Davis 1999). Each rotor spins the sample from each separate well, eliminating the need to transfer the plate to a centrifuge. The RevPrep isolates $5-10 \mu \mathrm{g}$ of plasmid DNA with a purity (260/280 ratio) of 1.8 from a 300- $\mu \mathrm{L}$ E. coli culture growth. A 96-well microplate of plasmid DNA can be isolated in $30 \mathrm{~min}$ at a cost of less than $\$ 0.10 /$ prep (S. Hunicke-Smith, pers. comm.). Up to 16 sample plates can be processed unattended in 8 hours. The template preparation procedure has performed well for the production of samples for capillary electrophoresis. The capillary instruments are particularly sensitive to impurities in the template preparations and therefore other cruder methods are not suitable (R. Gibbs, pers. comm.).

MWG Biotech, Inc. (http://www.mwgbiotech.com) has a new RoboPrep 2500 Automated Biosystem for automatic purification of nucleic acids such as isolation of plasmid DNA from E. coli cells and purification of PCR products. The system can process eight plates in seven hours for plasmid DNA or 2304 samples in 24 hours. A separate vacuum manifold is uded to eliminate contamination.

In June 2000, Applied Biosystems (http:// www.pebiosystems.com) introduced the ABI PRISM 6700 Automated Nucleic Acid Workstation that automates nucleic acid purification and reaction tray setup using a vacuum driven, flow-through membrane system that captures and washes the nucleic acids. Samples in a 96-well microplate are processed and capped with an optical cover in 90 minutes and are then ready for analysis in the ABI PRISM 7700.

At the Institute of Physical and Chemical Research (RIKEN) (http://genome.rtc.riken.go.jp/), in Tsukuba 
and Wako, Japan [along with the Japan Science and Technology Corporation (JST), (http://wwwalis.tokyo.jst.go.jp/HGS/top.html)], an automated high-throughput plasmid preparation system has been developed to process 40,000 samples in $17.5 \mathrm{hr}$. The samples can be used for restriction enzyme digests, DNA sequencing, and transcriptional sequencing. The system is based on the modified-filtration method (Itoh et al. 1997). Cell harvesting, alkaline lysis, and plasmid purification are performed on a single 96-well microplate, followed by collection of the sequenceready DNA samples with elution buffer. The plates are designed to allow for injection of all reagents from the top side of the 96 columns and the spent reagents vacuumed from the bottom side of the plates. This design enables a linear process for the plasmid preparation system. The system has an automated filter plate stacker and an automated plasmid preparator with 21 stages. At specific stages, various reagents or other chemistry are injected into the plates. (M. Itoh and Y. Hayashizaki, pers. comm.; Itoh et al. 1999).

Robbins Scientific Corporation's (Sunnyvale, CA) (http://www.robsci.com) Hydra Microdispensers are used at the WashU GSC in template purification and DNA sequencing reaction tasks to increase efficiency and reduce sample-to-sample variability (Mardis et al. 1997). Hydra-96 Channel Microdispensers equipped with $290-\mu \mathrm{L}$ syringes are used to dispense the reagents used in isolating plasmid DNA into 2-mL Beckman deep-well boxes (E. Mardis, pers. comm.). A method for purification of double-stranded plasmid DNA clones is described in Marra et al. (1999) and used at the WashU GSC to generate more than 700,000 ESTs at a rate exceeding 16,000/week.

Two robotic workstations for template purification are in regular production use at the Whitehead Institute/MIT Center for Genome Research (WICGR) (Cambridge, MA) (http://www-genome.wi.mit.edu). These were developed in collaboration with CCS/Packard (a custom automation company in Torrance, CA). The systems utilize 96-tip gantry pipettors and large linear tracks feeding plates to components such as plate stackers. Gantry pick and place robots move plates to orbital shakers and magnetic separators, plate washers, and plate sealers in various configurations. These workstations can purify M13 (60 plates/hr), plasmids (40 plates/hr), or PCR products (60 plates/hr). They use the SPRI (solid-phase reversible immobilization) protocol (Hawkins et al. 1994; DeAngelis et al. 1995). This procedure utilizes carboxylate-coated paramagnetic beads that exhibit a reversible affinity to precipitated DNA. The use of the paramagnetic beads as a solid support for the phage DNA coupled with a magnetic field greatly facilitate automation by eliminating difficult to automate centrifugation/filtration steps associated with most template purification protocols. A vacuum is required. The materials cost of the prep is $\$ 0.07 /$ sample including all reagents, plastics, and beads. The prep has proven to be reproducible and robust, giving sequencing pass rates of $92 \%$ (pass is assessed as read > 300-bp, phred 15) (P. McEwan, pers. comm.).

The section on Multi-Purpose Automated Workstations describes additional instruments capable of various DNA template preparation methods.

\section{Sequencing Reaction Robotics}

The Sequencing Support System (SSS), originally developed by H. Garner (Brignac 1997; Garner 1997), is an autonomous robot system for processing DNA sequence samples. The system is fully capable of dye terminator sequencing reactions and dye primer reactions. Integrated into the system are a $3 \mathrm{~m}$ Beckman Sagian (Beckman Coulter, Fullerton, CA) ORCA robot arm, two Robbins Scientific Hydra-96 pipettors, eight MJ Research (Waltham, MA) PTC-200 96-well thermal cyclers (expandable to four Tetrads), custom refrigerated plate hotels, and temperature controlled plate reservoirs. The system runs under Microsoft Windows95based software and has custom hardware drivers. The system generates eight consolidated 96-well dye primer plates in an 8-hr period and uses a text-pager to notify the user of impending needs. The plates are delivered to the operator ready for centrifugation. Another robotic system based on the Beckman Sagian ORCA is being developed to facilitate continuous and autonomous operation of the Molecular Dynamics MegaBACE 1000 DNA sequencer (T. Major, pers. comm.).

At the Baylor College of Medicine-Human Genome Sequencing Center (BCM-HGSC) (http:// www.hgsc.bcm.tmc.edu), in-house robot developments have focused on the development of a machine that carries out sequencing reactions. The system is based upon the SSS that was first constructed by Harold Garner at UTSW in Dallas, using a Beckman Sagian arm, Robbins Scientific HYDRA pipettors and MJ Research Thermocyclers. The BCM-HGSC device differs by the incorporation of a CRS Robotics Corp. robotic arm in place of the Sagian, the addition of a refrigerator containing a circular carousel, and the elimination of on-line thermocycling. A custom aluminum plate holder/sealer system has also been developed. These INGOTS eliminate the need for separate lids for each reagent or sample tray, and reduce evaporation of the very small volumes. The INGOTS can be stacked with as many as 30 plate sets (e.g. approximately 3000 DNA sequencing reactions) and the machine requires approximately five minutes to process each set which is the equivalent of more than 2 million reactions/yr. The BCM-HGSC version of the device is used in production to set up sequence reactions and to pool products (R. Gibbs, pers. comm.).

The MWG Biotech, Inc. RoboAmp 4200 robot (for 
automated non-cross-contamination PCR) and the RoboSeq 4200 robot (for sequencing reactions) both integrate an MWG-Biotech Primus thermocycler on-deck for a throughput of 600 sequence reactions in $8 \mathrm{hr}$ or up to 12 PCR plates unattended. To prevent cross contamination in the RoboAmp 4200, every vessel on the work surface is closed with airtight individual seals. Opening and closing of the special vessels takes place automatically with a novel sealing mechanism.

WICGR (Cambridge, MA) has three custom sequencing workstations (again developed in concert with CCS/Packard). The system elutes DNA from source plates, preps 384 forward and reverse sequencing reactions, and heat-seals the plates. Reactions are cooled by a recirculated refrigerant. The system preps $4-\mu \mathrm{L}$ reactions and can handle 26384 -well plates/hr. (P. McEwan, pers. comm.).

Dynal A.S. BioScience R\&D (Oslo, Norway) has developed an automated method based on biomagnetic separation technology using biotinylated sequencing primers and super-paramagnetic streptavidin beads. This method, which eliminates the centrifugation or filtration required for ethanol precipitation or column purification, removes excess dye terminators and other reagents from sequencing reactions. Sequencing reactions are performed with biotinylated primers and the biotinylated sequencing products are bound to streptavidin beads. The contaminants are washed away with $70 \%$ ethanol while the beads are held in a magnet. The method has been automated on robotic workstations to process 96 reactions in under one hr (Lukacs et al. 1999). See Schubert et al. (1999) for a similar approach.

\section{Thermal Cyclers}

Thermal cycling is an extremely important part of the large-scale DNA sequencing process, because of the need to perform PCRs and sequencing reactions. Traditionally, most cyclers take $2-4 \mathrm{hr}$ to perform the 30 or more cycles needed for PCR and sequencing reactions. Recent efforts have focused on scaling the sample volumes down, increasing the number of samples handled/run, and significantly decreasing the overall thermal cycling time required. A few systems are capable of cycling reactions in $20 \mathrm{~min}$.

GeneMachines is currently developing a 96channel thermal cycling device called the Tricycle (Hunicke-Smith and Batchelder 1998). It is estimated that $961-\mu \mathrm{L}$ samples will be amplified in the Tricycle system in about $30 \mathrm{~min}$ (S. Hunicke-Smith, pers. comm.).

The Idaho Technologies (Idaho Falls, ID) (http:// www.idahotech.com) RapidCycler is an air-based thermal cycler for 48 glass capillaries/run. Because of the reduced mass of the capillaries over conventional microplates and microtubes, cycling for PCR or sequencing reactions can be accomplished in as little as $20-30$ min (Wittwer et al. 1989; Wittwer and Garling 1991; Swerdlow et al. 1997).

Intelligent Bio-Instruments has a TC 1600 Thermocycler "waffle iron". It is a 16-block parallel thermal cycler for 96-, 192-, or 384-well microplates for up to 6144 samples/run. The thermocycler can run four sets of indepedendent heating and cooling protocols.

Andre Marziali at the University of British Columbia (Vancouver, BC, Canada), in collaboration with the Stanford DNA Sequencing and Technology Center, has developed an automated submicroliter thermal cycler, the T2, capable of processing $6 \times 96$ or 576 samples/ run at $500 \mathrm{~nL} /$ sample with $1 / 4$ diluted brews and $\$ 0.03 /$ sample for BigDye Terminator reactions. Samples are prepared in disposable tips, the ends sealed on a heat block, and then thermal cycled in a waterbased thermal cycler. Before samples are prepared inside the pipette tip, BSA is first aspirated in and out of the tip to prepare the surface. Samples and reagents are then added, with air separating each one. A water drop is also added to act as a vapor barrier. After thermal cycling, the melted tip ends are automatically snipped off and the samples are dispensed into 96-well microplates with $2 \mu \mathrm{L}$ of water so that the sample is not lost (A. Marziali, pers. comm.; http://www.physics.ubc.ca/ andre/).

In the Max-Planck-Institute (http://www.mpimgberlin-dahlem.mpg.de), large-scale DNA amplification is performed in the MPIMG water bath system under temperature control. Three heated 225-liter water baths house up to 135 plates $(51,840$ reactions) at one time. The basket of plates is moved from bath to bath using a pneumatic X-Z slide. The 384-well plates used for thermocycling are sealed with two-sided plastic film and a heat sealer. After cycling, the plastic film is easily removed and the samples are ready to be spotted directly onto nylon membranes for hybridization. A smaller thermocycler based upon this design was recently developed to house ten 384-well plates at a time for template preparation and cycle sequencing (Maier et al. 1997).

MJ Research, Inc. (http://www.mjr.com) has a line of Peltier-effect thermal cyclers: the PTC-200 DNA Engine, PTC-225 Tetrad, PTC-100, MiniCycler, In Situ Cycler, and MML-0150 Mobile Molecular Laboratory. The PTC-225 has four times (eight blocks) the capacity of the PTC-200 in a footprint just twice the size, can accommodate 384-well plates, can run two different protocols simultaneously on independently dual blocks, and has ramp rates of up to $3.0 \mathrm{deg}$.C/sec. The Mobile Molecular Laboratory is an entire laboratory for DNA analysis, including a MiniCycler, microcentrifuge, mini gel box, compact power supply, compact UV transilluminator, and benchtop enzyme chiller, packed into a suitcase for use anywhere in the world.

MWG Biotech has an extensive line of thermal cy- 
clers. The Primus 25 Personal Thermal Cycler System with $250^{\circ} \mathrm{C}$ HTR (High Temperature Range) Peltier technology $\left(-50-200^{\circ} \mathrm{C}\right)$ ahs heating rates up to $4^{\circ} \mathrm{C} /$ sec, integrated heated lids, and an exchangeable block system $(25 \times 0.2 \mathrm{~mL}, 13 \times 0.5 \mathrm{~mL}$ vessels, or $5 \times 5$ microplates). The Primus $96 \mathrm{HPL}$ and Primus $384 \mathrm{HPL}$ have High Pressure Lids (HPL) as well as the HTR Peltier technology. The HPL is a chemically actuated lid that provides up to $300 \mathrm{~N}$ of pressure to seal all PCR plates. A motorized lid is an option. For fast heating and cooling, the Primus in situ cycler has a flat, low-weight aluminum heating block that provides a capacity of four slides with up to three samples per slide. A gas tight reaction chamber with buffer reservoir minimizes evaporation. For high-throughput applications, the Primus Multiblock with HTR Peltier technology permits from 4-28 blocks, in sets of four, to be cycled simultaneously. Each block can hold 96- or 384-well plates with motorized HPLs.

The Applied Biosystems GeneAmp PCR System 9700 is a peltier-based thermal cycler with a heated cover that is available in 3 formats: 60 -well $0.5 \mathrm{~mL}$ tube format, 96-well $0.2 \mathrm{ml}$ format or a dual 384-well microplate format (768 wells total). PE Biosystems also has the ABI PRISM 877 (formerly the Catalyst) integrated thermal cycler that automates and integrates pipetting, PCR amplification, and pre- and post-PCR sample handling. It automatically pools the products for sequencing reactions. Liquid delivery and aspiration is performed with $100-\mu \mathrm{L}$ and 5 -mL glass syringes. A robotic arm positions a single steel probe with capacitive liquid sensing capability.

The RIKEN (http://genome.rtc.riken.go.jp) GS384 thermal cycler can process plates with 384 wells or 1536 wells. Plates can be automatically moved using a robotic arm. To achieve accurate temperature control with high thermo-condictivity, Teflon-coated aluminum well plates are closely sandwiched between a silicon-sheet-covered lid on top and a graphite sheet below. The lids are kept at a higher temperature (by 2-5 ${ }^{\circ} \mathrm{C}$ ) than the reaction wells. Temperature variability between wells and sample evaporation are minimal for samples as small as $2 \mu \mathrm{L}$ (Sasaki et al. 1998; Y. Hayashizaki, pers. comm.).

Robbins Scientific Corporation TruTemp DNA Microheating System accommodates 96 PCR tubes or 96well microplates. It has low mass resistance heaters around the chamber and also in the lid.

Stratagene (La Jolla, CA) (http://www.stratagene.com) temperature cyclers have four programmable thermal blocks; three are set individually for PCR steps and the fourth is cooled for sample storage. A highspeed robotic arm moves samples from block to block in under three seconds. The models include the RoboCycler Infinity for 40-, 96-, or 384-well blocks; the RoboCycler 40 and 96 for 40- or 96-well formats; and the
RoboCycler Gradient for 40- or 96-well formats, with a gradient feature that permits testing up to 12 PCR annealing temperatures in one run to optimize the annealing temperature.

Techne Inc. (Princeton, NJ) (http:// www.techneuk.co.uk) Progene thermal cycler for 25 micro tubes or 20 microcentrifuge tubes and the Techne Genius thermal cycler can also accommodate 96- and 384-well microplates. The new Techne Touchgene thermal cycler has a touch-sensitive screen for a user-friendly programming interface.

At the University of Washington, N. Friedman and D. Meldrum (Friedman and Meldrum 1998) developed a system that performs rapid thermal cycling of microliter and smaller liquid volumes inside glass capillary tubes having an optically transparent thin film of indium-tin oxide (ITO) covering the exterior. The ITO film acts as both a heater and temperature sensor, while cooling is accelerated with forced air. Temperature transition rates of $44^{\circ} \mathrm{C} / \mathrm{sec}$ during heating and $15^{\circ} \mathrm{C} / \mathrm{sec}$ during cooling have been achieved, allowing successful PCR experiments to be performed in $20 \mathrm{~min}$.

There are also thermal cyclers available for realtime quantitative PCR. These include the Idaho Technologies, Inc. LightCycler (now manufactured under license by Roche Molecular Biochemicals) and the Idaho Technologies, Inc. R.A.P.I.D. (Ruggedized Advanced Pathogen Identification Device), a fieldhardened rapid thermal cycler with fluorescence monitoring of 32 samples in glass capillaries based on the LightCycler. Applied Biosystems makes the ABI PRISM 7700 Sequence Detection System for quantitation of 96 samples/run, and Cepheid (Sunnyvale, CA) (http:// www.cepheid.com) makes the SmartCycler System for quantitation of 1-6 processing blocks with sixteen independently programmable reaction sites each and four-color realtime fluorometric detection. The Cepheid Smart Cycler XC is a battery operated version of the Smart Cycler. Cepheid also has the GeneXpert prototype that integrates rapid thermal cycling and realtime detection with a cartridge that performs sample preparation and DNA extraction including filtration, cell lysis, DNA extraction, and addition of PCR reagents.

\section{Multi-Purpose Automated Workstations}

Tremendous effort has gone into making fluid handling systems. Most of them use syringe-based pipetting methods, microplate formats, and handle typically $1 \mu \mathrm{L}$ sample volumes or more. With the recent trend toward miniaturization of processes in genome analysis, more systems are being developed that can handle submicroliter fluid volumes (e.g. Meldrum 1997 and Meldrum et al. 2000).

The Automation Partnership provides custom instrumentation services for customers. Their BaseLine 
system includes a range of hardware and software modules developed to provide flexible automation for genomic sample processing. The modules can operate as stand-alone units or be integrated into an automated processing facility. The module functions include picking, gridding, replicating, re-arraying, sequencing and PCR set-up, thermal cycling, plate storage and retrieval with sample tracking, microarraying, and gel loading. The BasePlate is a fully-automated robotic system with gridding and pipetting tools that handle liquid volumes from a few tens of picoliters to several hundred microliters. It can aspirate from 1-96 samples by pipette or by 384 pins and output in formats from a single well to a 1536 high-density array. The HomeBase is a compact, environmentally controlled plate store for genetics samples that is custom-made to the customers' specifications.

Beckman Coulter, Inc. (http://www.beckmancoulter.com) has provided a broad range of instruments for laboratory automation over the years. The earlier Beckman Biomek 1000 was used for automating dideoxynucleotide DNA sequencing reactions (Wilson et al. 1988; Zimmermann et al. 1988) and singlestranded DNA isolation (Mardis and Roe 1989). The Beckman Coulter Biomek 2000 Workstation is a general purpose modular platform controlled by the BioWorks software for pipetting, diluting, dispensing, and other options including heating and cooling, plate washing, high-density transfers, photometric measurement and high-capacity operation. Interchangeable pipette tools transfer from $1 \mu \mathrm{L}-1 \mathrm{~mL}$ and detection of liquids is possible with sonic liquid-sensing technology. An optional Side Loader System includes a robot arm and modular stack areas for high capacity, unattended operation. The Biomek system may be used for DNA sequencing reactions, DNA fingerprinting, PCR processes, DNA template preparation, library maintenance, and screening.

The newest liquid handling platform by Beckman Coulter, Inc. is the Biomek FX for nucleic acid preparation, plate replication, and screening. It has five times the deck space of the Biomek 2000. Applications include plasmid purification for both vacuum and magnetic bead-based protocols, PCR reaction setup and PCR or dye terminator cleanup. System flexibility is provided through the use of up to 24 automated labware positioners (ALPs) such as shakers, stirrers, tip washers, and heaters/coolers that can be stored or accessed from the deck as needed depending on the process being executed.

Another platform by Beckman Coulter, Inc. for sample preparation is the SAGIAN Core System that includes an ORCA robot on a one-, two-, or three-meter rail, Multimek 96 automated 96-channel pipettors, a Biomek 2000 or Biomek FX Laboratory Automation Workstation, development and scheduling software, and SAGIAN Stations such as thermal cyclers, incubators, microplate shakers, and so on. The Multimek 96 is a 96-channel automated pipettor that can transfer between 96- and 384-well microplates on the same work surface. It can be used for microplate replication, reagent addition, and full-plate serial dilutions.

The CRS Robotics Corporation DNA Amplification Workstation uses a CRS A465 robot, a Packard MultiPROBE II liquid handling workstation (see below), an MJR Thermocycler (see above), a Tomtec plate sealer, a refrigerated CRS carousel for microplates, a MicroScan barcode reader, and POLARA software to perform a complete amplification protocol.

GeneMachines works closely with the Stanford DNA Sequence and Technology Center headed by Dr. Ronald Davis (Marziali et al. 1999) to commercialize some of the technology that has been developed there for large-scale sequencing. In addition, it provides custom instrumentation for laboratories upon request. This may involve a complete custom design,or developing a combination of modules already available at GeneMachines to meet the required needs. Some of the instruments available at GeneMachines in their GeneSuite include the HydroShear (Thorstenson et al. 1998) to fragment DNA for shotgun sequencing; the Gel-2Well for colony/plaque picking; the HiGro unit that combines orbital shaking, oxygenation, and incubation for bacteria and phage growth of forty-eight 96well microplates/run; the Revolution PrepMachine for plasmid preparation using the Array Micro-Centrifuge that has 96 individual rotors into which one sample per each rotor can be automatically injected, spun down and retrieved; the PolyPlex for oligonucleotide synthesis; the OmniGrid for making microarrays; and the Automated Server Arm, a robot for transporting microplates between processing equipment.

In collaboration with CERMA, a French robotics company, Genoscope developed Autogel, a machine that uses thin agarose gels for separating pre-stained PCR products. The agarose gels are cast manually without sample wells in separate molding devices. The machine forms the wells and loads samples on the same robotic platform. The robot arm then moves the gel to an electrophoresis station that can simultaneously handle 16 gels. After electrophoresis, racks containing the gels are manually transferred to an imaging station where the gel images are taken automatically by a CCD camera and then downloaded to a database. With this robotic equipment they can analyze 15,000 -20000 PCR products with a size range of $100-600 \mathrm{bp}$, per day (G. Gyapay, pers. comm.).

The newest Hamilton Company (Reno, NV) (http://www.hamiltoncomp.com) robotic workstation is the MICROLAB 4000 series with multiple probe heads, single probes, solid phase extraction, and gridding with 96 or 384 pin formats. The MICROLAB 4000 
has a four-probe head and workspace for 18 microplates whereas the MICROLAB 4200 is available with 4 , $6,8,12$, or 96 probe heads and workspace for $38 \mathrm{mi}-$ croplates. The 96-probe head pipettes volumes from submicroliter to $500 \mu \mathrm{L}$ and has liquid level detection. These workstations can be configured as desired by the user for tasks such as solid phase extraction, DNA sequencing reactions, PCR preparations, and enzyme reaction setups. They may also be integrated with Zymark, CRS, Mitsubishi, or Sands Technology robotic arms.

Intelligent Bio-Instruments, in collaboration with Intelligent Automation Systems, Inc. provides custom instrumentation services for customers (Gordon et al. 1994). For example, the "Genomatron" was originally designed for use in the WICGR for high-throughput screening of libraries of DNA samples. The system includes a station for liquid handling and plate assembly with automatic in-line plate sealing, a massively parallel thermocycling station, and a vacuum blotting station that transfers reactions from thermocycling plates onto hybridization plates. Each station handles 96 custom made 1536-well plates for over 147,000 reactions/ run. Intelligent Bio-Instruments also has a handheld device called SPLATT, a Simultaneous Plate Loading and Transfer Tool. It can transfer liquids in volumes of $2-100 \mu \mathrm{L}$ from 96 wells at a time using any combination of 96-, 192-, or 384-well source and destination microplates.

MWG Biotech has a new RoboSNP 1500, a robotic system for automated PCR and processing of SNPs. The deck has four washable tip assemblies and four integrated 384-well HTR Primus thermal cyclers with motorized lids. The RoboSNP 1500 can be used with the Biflex III MALDI-TOF Mass Spectrometer (Bruker Daltonics, Inc.) for SNP detection and processing of 384 samples/hr.

Packard Instrument Company (Meriden, CT; http://www.packardinst.com) provides a line of MultiPROBE Robotic Liquid Handling Systems such as the MultiPROBE II and the MultiPROBE II HT. Applications include DNA extraction and plasmid preparation utilizing vacuum control, cycle sequencing reaction setups and thermal cycling. They can accommodate disposable tips and/or fixed washable tips with an eighttip capacity, have automatic Accusense liquid level sensing, Varispan variable spacing between sampling probes, and volume ranges from milliliters to nanoliters. The systems integrate with PlateStak microplate storage systems from CCS Packard, Inc. (http://www. ccspackard.com). The PlateStak and MiniTrak systems include MultiPosition Dispense (MPD) modules with three-axis motion, up to four microplate stackers for batch sizes up to 100 microplates, and pipetting with 96- or 384-channel heads. PlateStak can accommodate batch processing of 50-600 plates, and plate replica- tion and plate reformatting with 96-, 384-, or 1536well formats. MiniTrak has an open module for future expansions such as a wash module, vacuum filtration, lidding module or aspirator/dryer.

QIAGEN has a series of BioRobot Workstations. The new BioRobot 8000 workstations automate nucleic acid purification with an eight-channel pipetting system, a vacuum system to remove contaminants, and 384-well formats. For a variety of automated liquid handling and sample processing tasks, the BioRobot 3000 workstations can be configured as desired. These systems include robotic plate handling, highspeed pipetting with computer controlled high-precision peristaltic pump with solenoid valves for reagent delivery at up to $60 \mathrm{~mL} / \mathrm{minute}$, a variable temperature heating system and cooling system, shaker, vacuum manifold, and plate turntable. Applications include PCR setup and cleanup and nucleic acid purification. The BioRobot 9600 can process samples using one or two syringes with liquid level detection for DNA purification, reaction setups, rearraying samples, and gel loading.

Robbins Scientific Corporation systems include the Tango Liquid Handling System, Hydra Microdispensers, and Hydra Automated Dispensing Systems. The Tango includes quick-change glass syringe dispensing heads (96 and 384 syringes in $5 \mu \mathrm{L}, 100 \mu \mathrm{L}$, $290 \mu \mathrm{L}$, and $580 \mu \mathrm{L}$ syringe volumes), a disposable 96tip 200 ul volume syringe head, a 12-position X-Y stage for plate handling, and a 240-capacity plate stacker. The R/B (resin/bead) syringes may be used for dispensing magnetic beads or similar resins. Hydra Microdispensers are precision 96- and 384-channel dispensers for assembling reactions and dispensing template DNAs. They can be integrated into a variety of automated laboratory systems as described in various applications in this paper. Hydra Automated Dispensing Systems are automated plate handling accessories for the Hydra Microdispenser. They include an automated syringe washing system, an automated plate positioner, and a workstation that incorporates a 100-plate robotic plate handler with an automated plate positioning and syringe washing system.

The 290- $\mu \mathrm{L}$ syringe Hydras are used at the WashU GSC to dispense reagents for sequencing reactions into $0.2 \mathrm{~mL}$ thin wall PCR tubes. With the use of a removable plate-positioning device, they can use the Hydra96 for rapid, accurate dispensing of phage samples into 384-well microplates to reduce freezer storage space for long term storage. The Hydras are used for all pipetting done in the preparation and sequencing of over 250,000 templates/week. In addition, the Hydras have provided an increase in the overall quality of sequencing data and a reduction in pipetting errors over manual methods (Mardis et al. 1997; E. Mardis, pers. comm.).

Tecan (Research Triangle Park, NC) (http:// 
www.tecan-us.com) has a selection of workstations including the Genesis Robotic Sample Processor (RSP), the Genesis Robotic Microplate Processor (RMP), the MiniPrep 50 and MiniPrep 75, and the Tecan Robotic Assay Composer (TRAC). The systems are based upon Cavro (Sunnyvale, CA) syringe pumps and four or eight sampling tips on one arm. The RSP and RMP can accommodate a variety of modules and have a Robotic Manipulator (RoMa) arm to extend the range of operation. The GENESIS Workstation, designed to handle about 200 plates at a time, integrates a GENESIS RSP and a second arm with a five-point rotational gripper to move microplates. In late summer 2000, the workstation will include a 96-channel pipetting option called GenMate. Tecan also recently introduced a new nanoliter miniaturized assay workstation.

Tomtec (Hamden, CT) (http://www.tomtec.com) has a variety of liquid handling platforms for a broad range of applications. The Quadra line of automatic assay workstations include models for 96-well pipetting or 384-well pipetting along with X-Y-Z stages, plate stackers, and ultrasonic tip washers. The newest model is the Quadra 3 with interchangeable heads. Pipetting is with positive air displacement for volumes from $0.5 \mu \mathrm{L}$ to $450 \mu \mathrm{L}$ depending on the head. Multiple samples may be aspirated, using air gap separation, to complete reagent addition steps in one automated pass instead of multiple passes.

The Genomation Laboratory at the University of Washington (http://rcs.ee.washington.edu/GNL/ genomation.html) has been designing and building an automated, multi-purpose, submicroliter to microliter fluid handling system for processes such as restriction enzyme digests, PCRs, and sequencing reactions (Arutunian et al. 1998; Daoura and Meldrum 1999; Evensen et al. 1998; Friedman and Meldrum 1998; Meldrum et al. 2000; Sjoboen and Meldrum 1998). The system, called Acapella, processes 5000 glass capillaries in eight hours with one sample/capillary and total reaction volumes of 1-2 $\mu \mathrm{L}$. Modules for the system include a reaction preparation module for aspirating, piezoelectric dispensing and mixing of fluids; automated thermal cycling; and magnetic bead-based purification. With support of the NIH NHGRI the Acapella system will be alpha-tested with the University of Washington Genome Center (UWGC) (http:/www.genome.washington.edu/UWGC/) starting in December 2000 and betatested with the UWGC, the WashU GSC, and the WICGRI starting in June 2002.

In order to reduce sequencing production costs while simultaneously maintaining high quality standards, the WashU GSC (St. Louis, MO) developed a conveyor belt type robotic system called AutoPrep for DNA sample preparation. The first AutoPrep went in to use in September 1998 and four AutoPreps were in full production as of March 1999. The system automati- cally transfers M13 supernatant to PEG/NaClcontaining boxes and glycerol-containing archive plates. Pipetting steps are performed with Robbins Hydra Microdispensers. The AutoPrep system has a throughput of 12 boxes/40 min. It runs unattended, allows for recipe changes and adjustments in box/tray aspirate/dispense depths, has bar code reading, network interfacing, data logging, real-time reporting, and user intervention for fault recovery and run continuation (E. Mardis and D. Panussis, pers. comm.).

With a Zymark Robotic Workstation (Zymark Corporation, Hopkinton, MA) (http://www.zymark.com), the user can configure the system to include whatever modules are required for their needs. For example, an early Zymark system, built to perform 240 sequencing reactions in $7 \mathrm{hr}$, consists of a Zymate XP robot arm, a System V controller, and Pytechnology components (pipet-tip storage racks, microplate storage racks, pipettip workstation, microplate workstations and two "hands" to move objects) configured in a cylindrical work space (Earley 1994). More recent systems include the Presto Liquid Handler, RapidPlate 96 Pipetting Workstation, Benchmate II Workstations, and the Virtuoso Assay Automation Systems.

In August 1999, Zymark acquired Scitec Automation (Lausanne, Switzerland) and in May 2000 introduced the Staccato Workstation that includes a SciClone liquid handling device, a Presto autostacker, CLARA 2000 software, a Mitsubishi Model RV-1A sixaxis articulated robot arm with a microplate gripper head, optional readers, washers, incubators, and filtration units. Configurations of the workstations include the Staccato DNA Purification Workstation with a throughput of up to 10,000 plasmid DNA preps/day, the Staccato DNA Amplification Workstation, and the Staccato DNA Clean-up Workstation.

\section{Conclusions}

The development of new technology for genomics is an interdisciplinary effort requiring the collaborative efforts of molecular biologists, geneticists, chemists, physicists, mathematicians, computer scientists, and engineers (Meldrum 1995). By automating process steps with more conventional robots and instruments, great improvements are already being made in terms of throughput, efficiency, quality, reliability, and cost reduction. Large-scale sequencing efforts and genome analysis are just starting on a track of exponential growth in throughput. The second half of this review will focus on DNA sequencers, array technology, and future automation efforts.

\section{ACKNOWLEDGMENTS}

Deirdre Meldrum thanks the many people whose generous private communications made this article possible. 


\section{REFERENCES}

Arutunian, E.B., Meldrum, D.R., Friedman, N.A., and Moody, S.E. 1998. Flexible software architecture for user-interface and machine control in laboratory automation. Bio Techniques 25: 698-700, 702, 704-705.

Brignac, S. 1997. Sequencing support system: a robotic system for processing DNA samples. IEEE EMB 16: 92-93.

Burks, C. 1994. DNA sequence assembly. IEEE EMB 13: 771-773.

Collins, F.S., Patrinos, A., Jordan, E., Chakravarti, A., Gesteland, R. and Walters, L. 1998. New goals for the U.S. Human Genome Project. Science 282: 682-689.

Dahl, C.A. and Strausberg, R.L. 1996. Revolutionizing biology through leveraging technology. IEEE EMB 15: 106-110.

DeAngelis, M.M., Wang, D.G. and Hawkins, T.L. 1995. Solid-phase reversible immobilization for the isolation of PCR products. Nucleic Acids Res. 23: 4742-4743.

Daoura, M. and Meldrum, D. 1999. Precise automated control of fluid volumes inside glass capilaries. IEEE J. Microelect. Sys 8: $71-77$.

Earley, J.J., Kuivaniemi, H., Prockop, D.J., and Tromp, G. 1994 Robotic automation of dideoxyribonucleotide sequencing reactions. BioTechniques 17: 156-165.

Evensen, H.T., Meldrum, D.R., and Cunningham, D.L. 1998. Automated fluid mixing in glass capillaries. AIP Rev. Sci. Instr. 69: 519-526.

Friedman, N. and Meldrum, D. 1998. Capillary tube resistive thermal cycling. Anal. Chem. 70: 2997-3002.

Garner, H.R. 1997. Custom hardware and software for genome center operations: from robotic control to databases. In Automation Technologies for Genome Characterization (ed., T.J. Beugelsdijk), pp. 3-41. John Wiley \& Sons, Inc., New York.

Goodman, N. 1996. The fundamental principles for constructing a successful biological laboratory informatics system. Sci. Comput.Automation 8: 29-31,34-36. Also at http://www.asia-pacific.digital.com/info/onlinelab/whitepapers/ white-paper.htm.

Gordon, S.J., Christopher, A.J., and Valle, J.L. 1994. Engineering automated systems for physical mapping. IEEE EMB 13: $547-550$.

Hawkins, T.L., O'Connor-Morin, T., Roy, A., and Santillan, C. 1994. DNA purification and isolation using a solid-phase. Nucleic Acids Res. 22: 4543-4544.

Hawkins, T. L., McKernan, K.J., Jacotot, L.B., MacKenzie, J.B., Richardson, P.M., and Lander, E.S. 1997. A magnetic attraction to high-throughput genomics. Science 276: 1887-1889.

Hood, L.E., Hunkapiller, M.W., and Smith, L.M. 1987. Automated DNA sequencing and anlaysis of the human genome. Genomics 1: 201-212.

Hunicke-Smith, S.P. and Batchelder, K.A. 1998. A new device for thermal cycling of small volume PCR and sequencing reactions. Microb. Comp. Genom. 3: C-27.

Hunkapiller, T., Kaiser, R.J., Koop, B.K., and Hood, L. 1991 Large-scale and automated DNA sequence determination. Science 254: 59-67.

Itoh, M., Carninci, P., Nagaoka, S., Sasaki, N., Okazaki, Y., Ohsumi, T., Muramatsu, M., and Hayashizaki, Y. 1997. Simple and rapid preparation of plasmid template by a filtration method using microtiter filter plates. Nucleic Acids Res. 25: 1315-1316.

Itoh, M., Kitsunai, T., Akiyama, J., Shibata, K., Izawa, M., Kawai, J., Tomaru, Y., Carninci, P., Shibata, Y., Ozawa Y. et al. 1999. Automated filtration-based high-throughput plasmid preparation system. Genome Res. 9: 463-470.

Jandreski, M.A. 1995. Novel methods of DNA analysis. Clinics Lab. Med. 15: 817-837.

Lashkari, D.A., Hunicke-Smith, S.P., Norgren, R.M., Davis, R.W., and Brennan, T. 1995. An automated multiplex oligonucleotide synthesizer: Development of high-throughput, low-cost DNA synthesis. Proc. Nat. Acad. Sci. 92: 7912-7915.

Lukacs, M.F., Deggerdal, A.H., and Bosnes, M. 1999. Automated method for purification of Dye Terminator sequencing products. Microb. Comp. Genom. 4: 109.
Macilwain, C. 2000. World leaders heap praise on human genome landmark. Nature 405: 983-985.

Maier, E., Bancroft, D.R., and Lehrach, H. 1997. Large-scale library characterization. In Automation Technologies for Genome Characterization (ed. T.J. Beugelsdijk), pp. 65-88. John Wiley \& Sons, Inc., New York.

Maier, E., Meier-Ewert, S., Bancroft, D.R., and Lehrach, H. 1997. Automated array technologies for gene expression profiling. Drug Disc. Today 2: 315-324.

Mardis, E.R., Weinstock, L., Simonyan, A., and Stanchfield, J.E. 1997. M13 DNA preparations for a large scale sequencing project using the Hydra-96 channel microdispenser. Robbins Scientific Innovations 5: 1-4.

Marra, M.A., Kucaba, T.A., Hillier, L.W., and Waterston, R.H. 1999. High-throughput plasmid DNA purification for 3 cents per sample. Nucleic Acids Res. 27: e37.

Marziali, A., Willis, T.D., Federspiel, N.A., Davis, R.W. 1999. An automated sample preparation system for large-scale DNA sequencing. Genome Res. 9: 457-462.

Marziali, A., Willis, T.D., and Davis, R.W. 1999. An arrayable flow-through microcentrifuge for high-throughput instrumentation. Proc. Natl. Acad. Sci. 96: 61-66.

Maxam, A.M. and Gilbert, W. 1977. A new method for sequencing DNA. Proc. Natl. Acad. Sci. 74: 560-564.

Meldrum, D.R. 1995. The interdisciplinary nature of genomics. IEEE EMB 14: 443-448.

Meldrum, D.R. 1997. A biomechatronic fluid-sample-handling system for DNA processing. IEEE/ASME Transactions on Mechatronics 2: 99-109.

Meldrum, D.R., Evensen, H.T., Pence, W.H., Moody, S.E. Cunningham, D.L., and Wiktor, P. 2000. ACAPELLA-1K, a capillary-based submicroliter automated fluid handling system for genome analysis. Genome Res. 10: 95-104.

Mullikin, J.C. and McMurragy, A.A. 1999. Techview: DNA sequencing. Sequencing the genome, fast. Science 283: $1867-1869$.

Mullis, K.B., Faloona, F.A., Scharf, S.J., Saiki, R.K., Horn, G.T., and Erlich, H.A. 1986. Specific enzymatic amplification of DNA in vitro: the polymerase chain reaction. Cold Spring Harbor Symp. Quant. Biol. 51: 263-273.

Mullis, K.B. and Faloona, F.A. 1987. Specific synthesis of DNA in vitro via a polymerase-catalyzed chain reaction. Meth. Enzymol. 155: $335-350$.

Panussis, D.A., Stuebe, E.T., Weinstock, L.A., Wilson, R.K., and Mardis, E.R. 1996. Automated plaque picking and arraying on a robotic system equipped with a CCD camera and a sampling device using intramedic tubing. Lab. Robot. Autom. 8: 195-203.

Rayner, S., Brignac, S., Bumeister, R., Belosludtsev, Y., Ward, T., Grant, O., O'Brien, K., Evans, G.A., and Garner, H.R. 1998. MerMade: An oligodeoxyribonucleotide synthesizer for high-throughput oligonucleotide production in dual 96-well plates. Genome Res. 8: 741-747.

Roberts, S.L. 1993. Taking stock of the genome project. Science 262: 22-22.

Sanger, F., Nicklen, S., and Coulson, A.R. 1977. DNA sequencing with chain-terminating inhibitors. Proc. Natl. Acad. Sci. 74: $5463-5467$.

Sasaki, N., Izawa, M., Watahiki, M., Ozawa, K., Tanaka, T., Oneda, Y., Matsuura, S., Carninci, P., Muramatsu, M., Okazaki, Y. et al. 1998. Transcriptional sequencing: a method for DNA sequencing using RNA polymerase. Proc. Natl. Acad. Sci. 95: 3455-3460.

Sasaki, N., Nagaoka, S., Itoh, M., Izawa, M., Konno, H., Carninci, P., Yoshiki, A., Kusakabe, M., Moriuchi, T., Muramatsu, M. et al. 1998. Characterization of gene expression in mouse blastocyst using single-pass sequencing of 3995 clones. Genomics 49: $167-179$.

Schubert, F., Zimmermann, W., and Wambutt. R. 1999. Dye terminator removal and sequencing reaction clean up utilization of superparamagnetic particles. Microb. Comp. Genom. 4: 114 .

Scientific American. July 2000, Special Report: The Human Genome Business. 
Sindelar, L.E. and Jaklevic, J.M. 1995. High-throughput DNA synthesis in a multichannel format. Nucleic Acids Res. 23: 82-987.

Sjoboen, L. and Meldrum, D. 1998. Compact jam resistant capillary dispenser for automation systems. Lab Rob. Autom. 10: 15-17.

Smith, L.M., Fung, S., Hunkapiller, M.W., Hunkapiller, T.J., and Hood, L. 1985. The synthesis of oligonucleotides containing an aliphatic amino group at the 5' terminus: synthesis of fluorescent DNA primers for use in DNA sequence analysis. Nucleic Acids Res. 13: 2399-2412.

Smith, L.M., Sanders, J.Z., Kaiser, R.J., Hughes, P., Dodd, C., Connell, C.R., Heiner, C., Kent, S.B.H., and Hood, L.E. 1986. Fluorescence detection in automated DNA sequence analysis. Nature 321: $674-679$.

Smith, T.M., Abajian, C., and Hood, L. 1997. Hopper: software for automating data tracking and flow in DNA sequencing. CABIOS 13: $175-182$.

Spurr, N., Darvasi, A., Terrett, J., and Jazwinska, L. 1999. New technologies and DNA resources for high throughput biology. $\mathrm{Br}$. Med. Bull. 55: 309-324.

Swanson, D. 2000. The art of the state of nucleic acid sequencing: highly refined automated sequencing systems keep up with demand for large-scale genomic projects. The Scientist 14: 23.

Swerdlow, H., Jones, B.J., and Wittwer, C.T. 1997. Fully automated DNA reaction and analysis in a fluidic capillary instrument. Anal. Chem. 69: 848-855.

Thorstenson, Y.R., Hunicke-Smith, S.P., Oefner, P.J., and Davis, R.W 1998. An automated hydrodynamic process for controlled, unbiased DNA shearing. Genome Res. 8: 848-855.

Uber, D.C., Jaklevic, J.M., Theil, E.H., Lishanskaya, A., and McNeely, M.R. 1991. Application of robotic and image processing to automated colony picking and arraying. BioTechniques 11: $642-647$.

Venter, J.C., Adams, M.D., Sutton, G.G., Kerlavage, A.R., Smith, H.O., and Hunkapiller, M. 1998. Shotgun sequencing of the human genome. Science 280: 1540-1542.

Venter, J.C., Smith, H.O., and Hood, L. 1996. A new strategy for genome sequencing. Nature 381: 364-366.

Waterston, R. and Sulston, J.E. 1998. The Human Genome Project: reaching the finish line. Science 282: 53-54.

Watson, A., Smaldon, N., Lucke, R., and Hawkins, T. 1993. The Caenorhabditis elegans genome sequencing project: first steps in automation. Nature 362: 569-570.

Wendl, M.C., Dear, S., Hodgson, D., and Hillier, L. 1998. Automated sequence preprocessing in a large-scale sequencing environment. Genome Res. 8: 975-984.

White, O. and Kerlavage, A.R. 1996. TDB: new databases for biological discovery. Meth. Enzym. 266: 27-40.

Wittwer, C.T., Fillmore, G.C., and Hillyard, D.R. 1989. Automated polymerase chain reaction in capillary tubes with hot air. Nucleic Acids Res. 17: 4353-4357.

Wittwer, C.T. and Garling, D.J. 1991. Rapid cycle DNA amplification: time and temperature optimization. BioTechniques $10: 76-83$.

Zhang, J.Z., Fang, Y., Hou, J.Y., Ren, H.J., Jiang, R., Roos, P., and Dovichi, N.J. 1995. Use of non-cross-linked polyacrylamide for four-color DNA sequencing by capillary electrophoresis separation of fragments up to 640 bases in length in two hours. Anal. Chem. 67: 4589-4593.

Zimmermann, J., Voss, H., Schwager, C., Stegemann, J., and Ansorge, W. 1988. Automated Sanger dideoxy sequencing reaction protocol. FEBS Letters 233: 432-436. 


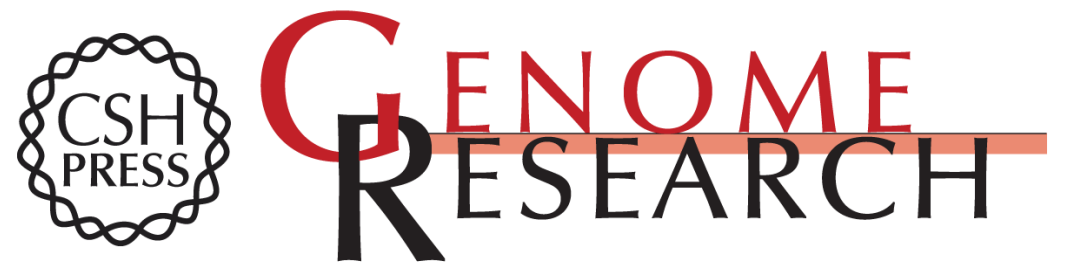

\section{Automation for Genomics, Part One: Preparation for Sequencing}

Deirdre Meldrum

Genome Res. 2000 10: 1081-1092

Access the most recent version at doi:10.1101/gr.101400

References This article cites 52 articles, 18 of which can be accessed free at:

http://genome.cshlp.org/content/10/8/1081.full.html\#ref-list-1

License

Email Alerting Receive free email alerts when new articles cite this article - sign up in the box at the Service top right corner of the article or click here.

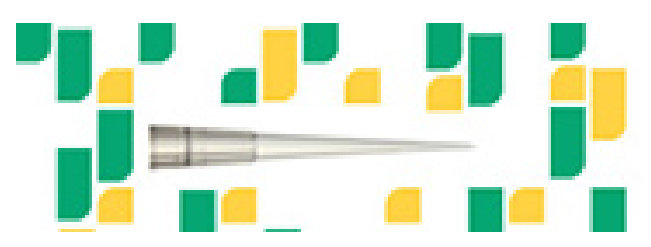

To subscribe to Genome Research go to: https://genome.cshlp.org/subscriptions 